\title{
Evaluation of Paint Coating on Metal Elements Using Scanning Pulsed Eddy Current Thermography
}

\author{
by W. Swiderski*, P. Hlosta
}

*Military Institute of Armament Technology, Wyszynskiego 7, 05-220, Zielonka, Poland, waldemar.swiderski@wp.pl

\begin{abstract}
The paper presents an active thermography method that utilizes eddy current heating to evaluate the uniformity of the corrosion protection layer on a metal element. Eddy currents, also known as Foucault currents, have been known for several decades and serve as a method of non-destructive testing. The new method uses eddy current for thermal stimulation of the test material [1]. Pulsed Eddy Current Thermography is a technique that uses pulses of eddy currents induced in conducting media to generate local heating inside the material. The transient diffusion of the heat inside the material, induced by pulsed induction heating, is imaged by measuring the transient temperature profiles on the surface of the material [2]. The presence and characteristics of the defects inside the materials changes the surface temperature transients and thus can be used for the nondestructive evaluation (NDE) of conducting materials [3, 4]. Experimental tests demonstrated that this method is capable of detecting non-uniformity in very thin layers of corrosion protection of flat metal elements.
\end{abstract}

\section{Introduction}

One of the most commonly used methods of corrosion protection for metal parts is the application of paints and varnishes. In order for the protective coating to function properly, it must be applied to the entire surface of a metal element with uniform thickness. Areas where the coating is thinner than required are exposed to corrosion in less time than those covered by the required coating thickness. Therefore, it is important to evaluate uniformity of the protective layer application. Assessment of the application uniformity of a layer of paint should take place both prior to the period of use as well as during use. During operation, corrosion may occur, which may not be visible because it will occur beneath a layer of paint. For such an assessment paint coating are the most effective non-destructive testing methods. One such method is infrared thermography with thermal stimulation of the test object by eddy currents.

\section{Pulsed Eddy Current Thermography}

Non-destructive testing methods using infrared thermography are based on detection of defects in the material and provide information about properties of the object being examined without depriving it of utility values. Methods of active infrared thermography require use of a source of thermal stimulation of the tested object. One active thermographic method is pulsed eddy current thermography, in which eddy currents are used as a heat source. It is a relatively safe method for tested objects because temperature increase on its surface does not usually exceed more than several degrees. Eddy current thermography combines NDT thermography and eddy current techniques, providing a fast and effective method for detecting and characterizing defects on relatively large surfaces. Thermographic data and images can be immediately evaluated in order to identify major defects. Eddy current thermography requires the use of high frequency eddy currents (usually from several dozen to several hundred $\mathrm{kHz}$ ). In the case of the pulsed method, eddy currents are generated for a short period of time (usually $20 \mathrm{~ms}-2 \mathrm{~s}$ ) [5]. Induced eddy currents are converted into heat by ohmic heating, in accordance with Joule's law (1).

$$
Q=I \cdot R^{2} \cdot t
$$

where

Q - amount of heat generated;

I - intensity of electric current;

$\mathrm{R}$ - electrical resistance conductor;

$\mathrm{t}$ - time of current flow.

Induction heating is carried out via an electromagnetic coil using alternating current at a specific frequency. The induction coil is placed near the surface of the test sample. This method can be used not only for non-destructive testing to detect defects but also as a means to identify the characteristics of materials for measuring thermal diffusivity and electrical conductivity. 


\section{Modeling and Simulation}

In order to assess the possibility of using pulsed eddy current thermography and to determine the thermal excitation parameters of eddy currents, ThermoEdCur software was used to assess homogeneity of paint coating on metal elements. This is a program developed by Professor Vavilov (Tomsk University) and carried out at MIAT.

ThermoEdCur software is designed to calculate three-dimensional temperature distributions in anisotropic multilayer solids that may contain subsurface defects. The appropriate mathematical problem of heat conduction is modeled in Cartesian coordinates and solved using the default numerical scheme with a finite difference. The unique numerical algorithm used in ThermoEdCur, unlike many commercial programs available, enables modeling very thin defects in rather thick materials without losing computational accuracy. It enables defect analysis when the sample is heated uniformly or non-uniformly with a square or cosine pulse. A unique feature of the program is that the heating can be performed by a moving heat source, thus modeling infrared (IR) thermography with eddy current.

The tested model is heated on the front surface by means of an external heat source (eddy current generator). It is assumed that the heat flux on the face moves at a constant speed. Together with the external thermal stimulation on the front surface, both the front and back surfaces are cooled according to Newton's law (according to this law, both convection and radiant heat exchange mechanisms are combined and described with a specific value of heat exchange coefficient). The thermal properties of the samples and any defects can be determined separately in three spatial directions, thus modeling the completely anisotropic material. The specimen side surfaces are adiabatic and on the boundaries between the specimen layers and between the host materials and the defects, the temperature and heat flux continuity conditions occur. In ThermoEdCur, the concept is of the so-called capacitive defects which means that, unlike resistive defects associated with some other NDT models, both thermal defects and conductivity are used in the calculations. This provides the most correct description of physical phenomena occurring in defect areas [6].

In the case of heating with eddy currents, the stimulating thermal energy penetrates deeply, where the attenuation is described by the following formula [7]

$$
d Q=Q_{0} e^{\gamma \cdot d z}
$$

where $d Q$ is the energy absorbed within the $d z$ distance, and $\gamma$ is the eddy current absorption coefficient defined by:

$$
\gamma=\frac{\sqrt{\pi f \sigma \mu_{0} \mu}}{2}
$$

Here $f$ is the eddy current frequency, $\sigma$ is the electrical conductivity $[\mathrm{S} / \mathrm{m}], \mu_{0}=1.257 \cdot 10^{-6} \mathrm{~N} / \mathrm{m}$ is the magnetic permeability of free space $[\mathrm{H} / \mathrm{m}], \mu$ is the material relative magnetic permeability. Therefore, eddy current energy absorption is characterized by the following parameters: $f, \sigma, \mu$.

A linear heat source (Fig.1) with an even energy distribution, starts at the edge of the sample to move on the surface of the sample at a constant velocity $V$. Source movement can be performed along any $X$ and $Y$ surface coordinate. In this case, the result of the calculation is a synthetic image in which each of the following lines is recorded at a fixed distance from the back edge of the heating line. In this way, the resulting image corresponds to a particular time delay. 


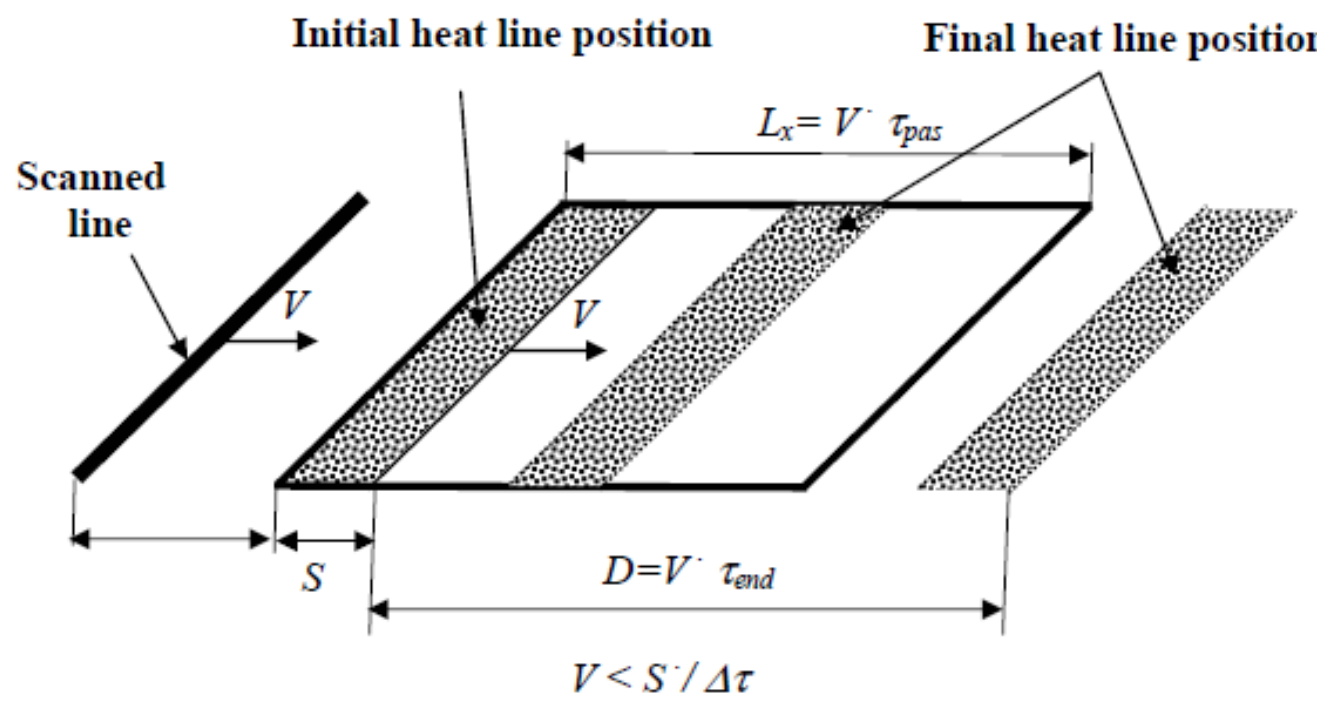

Fig. 1. Heating a sample with a heating line

In order to simulate the possibility of assessing the effect of the thickness of the paint layer covering the surface of metal, samples with different thickness of the paint layer and samples with defects on the surface of the material covered with the paint layer were analyzed. The thermophysical properties of metals and paint were adopted on the basis of literature data $[5,7]$ and the thermophysical properties of a camouflage coating paint (brand name: EC Paint) applied in thin layers [8]. Thin layer EC Paint exhibits the properties of a typical insulator, with density of about $1485 \mathrm{~kg} \cdot \mathrm{m}^{-3}, \mathrm{specific}$ heat equal to $1273 \mathrm{~J} \cdot \mathrm{kg}^{-1} \cdot \mathrm{K}^{-1}$, and thermal conductivity equal to $0.254 \mathrm{~W} \cdot \mathrm{m}^{-1} \cdot \mathrm{K}^{-1}$ at about $30^{\circ} \mathrm{C}$. For metals, the parameters adopted are presented in Table 1.

Table 1. Thermophysical properties of metals [5,7]

\begin{tabular}{|l|c|c|c|c|c|}
\hline Material & $\begin{array}{c}\text { Specific heat } \\
{[\mathrm{J} / \mathrm{kg} \cdot \mathrm{K}]}\end{array}$ & $\begin{array}{c}\text { Thermal } \\
\text { conductivity } \\
{[\mathrm{W} / \mathrm{m} \cdot \mathrm{K}]}\end{array}$ & $\begin{array}{c}\text { Density } \\
{\left[\mathrm{kg} / \mathrm{m}^{3}\right]}\end{array}$ & $\begin{array}{c}\text { Electrical } \\
\text { conductivity } \\
{[\mathrm{S} / \mathrm{m}]}\end{array}$ & $\begin{array}{c}\text { Relative } \\
\text { magnetic } \\
\text { permeability }\end{array}$ \\
\hline Steel & 434 & 63.9 & 7830 & $1 \cdot 10^{7}$ & 5000 \\
\hline Aluminum & 765 & 46 & 3970 & $3.5 \cdot 10^{7}$ & 1.000022 \\
\hline Copper & 406 & 365 & 9000 & $5.96 \cdot 10^{7}$ & 0.999994 \\
\hline
\end{tabular}

Figure 2 presents the tested model of a metal sample (thickness of $2 \mathrm{~mm}$ and dimensions of $40 \times 140 \mathrm{~mm}$ ) covered with paint layers. $1 / 3$ of the sample surface with one-, two- and three-layers of paint respectively. It was assumed that a single layer of paint has a thickness of about $80 \mu \mathrm{m}$, a double layer $160 \mu \mathrm{m}$, and a triple layer $240 \mu \mathrm{m}$. Figures 3-5 present the results obtained after simulating the heating of samples by eddy current made of metals whose parameters are shown in Table 1. The graphs show temperature changes on the surface of the samples from the heating side. The speed of movement of the heating line was $V=3 \mathrm{~cm} / \mathrm{s}$ and the heating power $\mathrm{Q}=3 \cdot 10^{4} \mathrm{~W} / \mathrm{m}^{2}$.

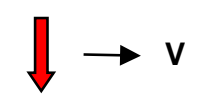

Fig. 2. Model of a metal sample covered with paint layers 


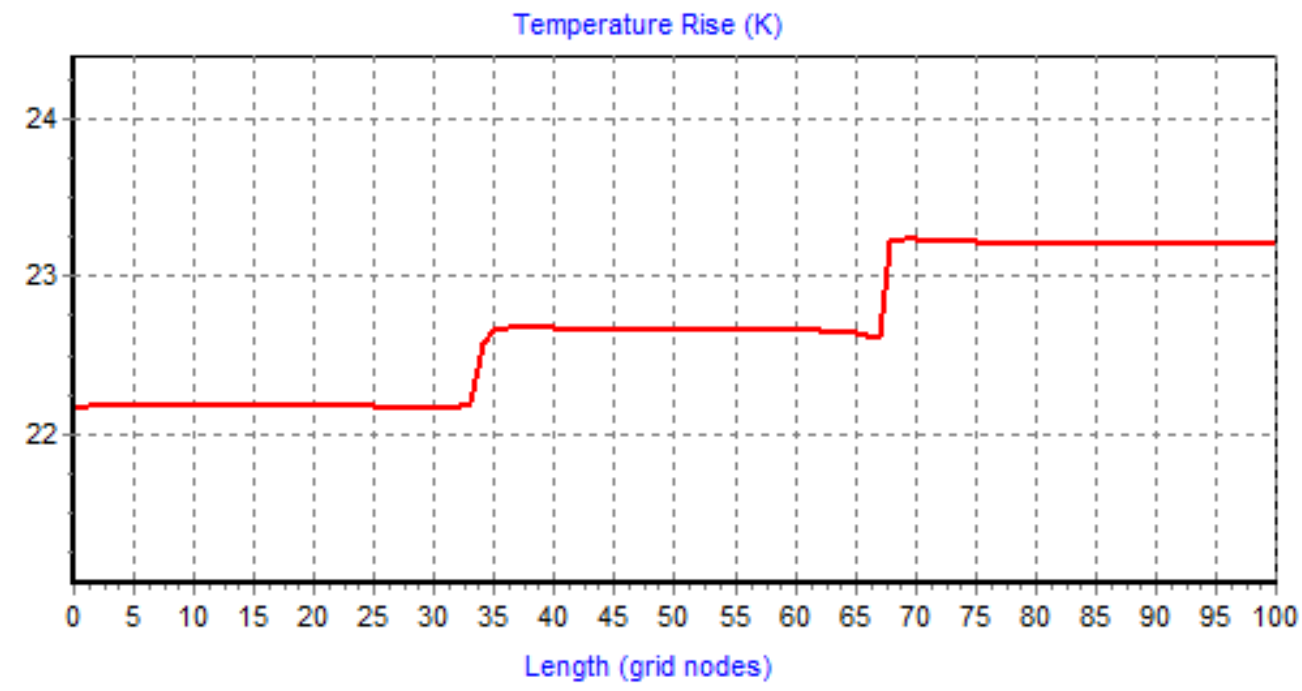

Fig. 3. Temperature changes on the surface of steel samples from the heating side.

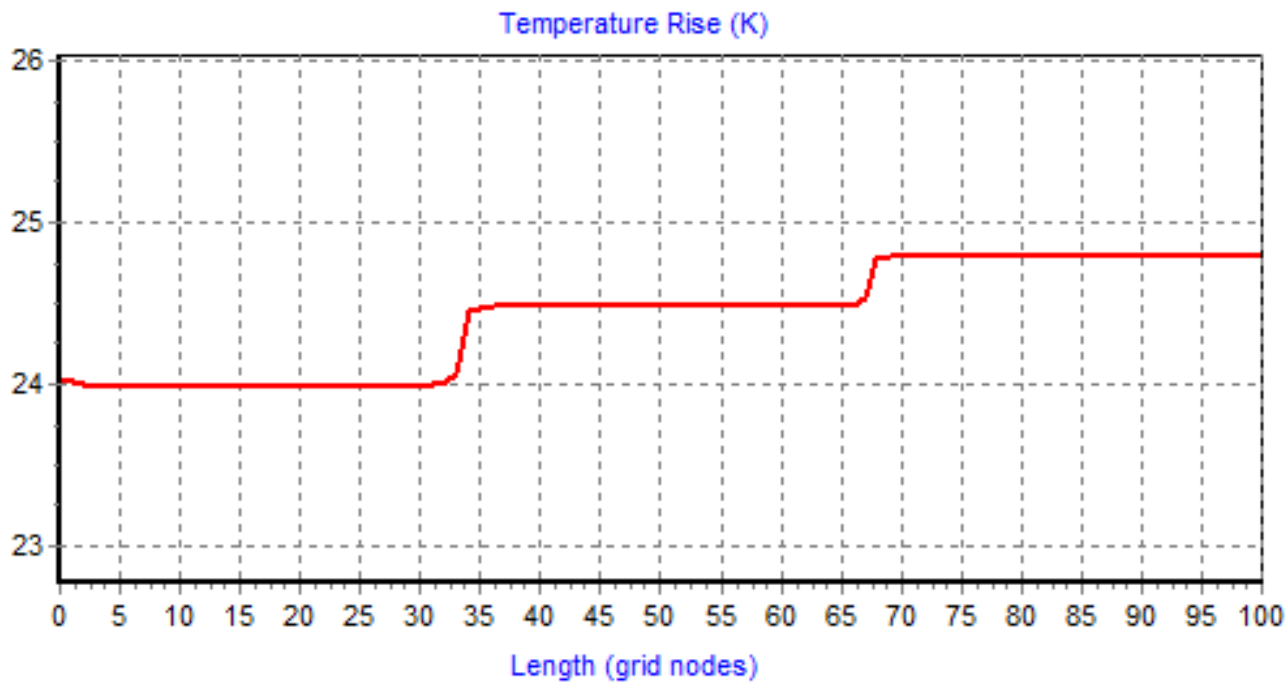

Fig. 4. Temperature changes on the surface of aluminum sample from the heating side 


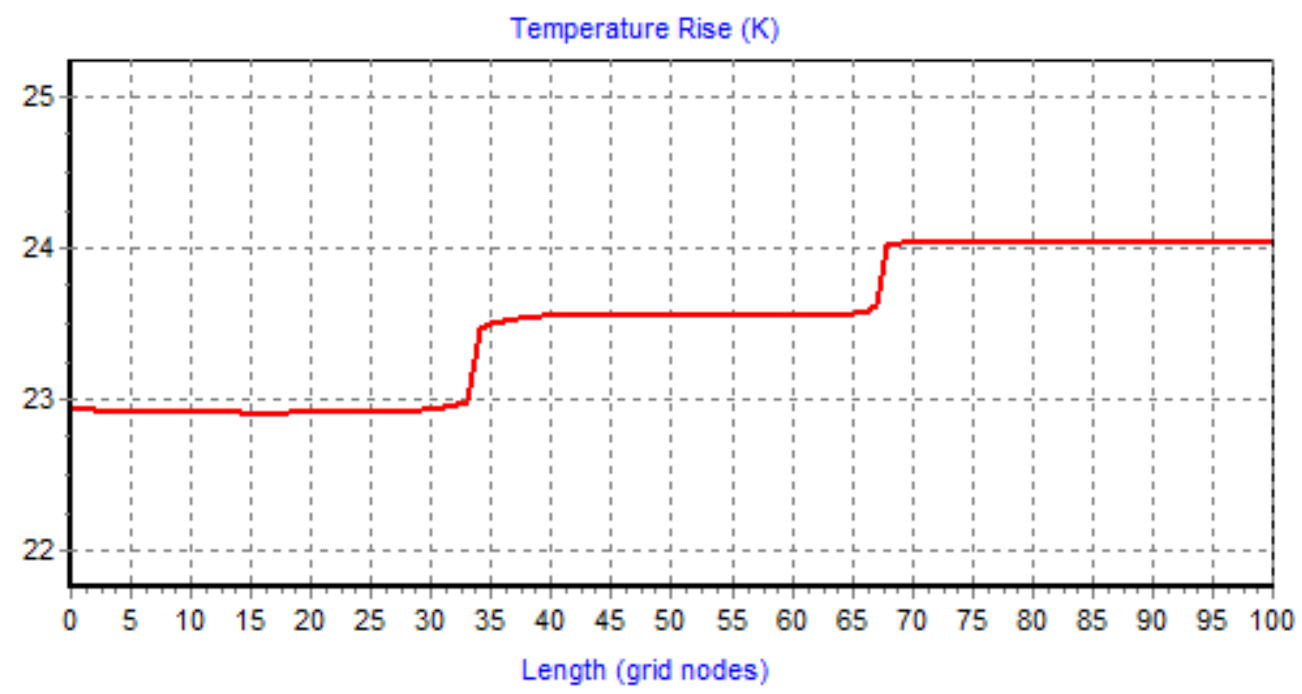

Fig. 5. Temperature changes on the surface of copper sample from the heating side

\section{Experimental Testing}

Experimental tests were carried out on three flat samples with a thickness of $2 \mathrm{~mm}$ and dimensions of $40 \times 140$ $\mathrm{mm}$ composed of various metals (steel, aluminum and brass). The samples were covered in $1 / 3$ of their length with different thicknesses of paints representing respectively one-, two-, and three layers. The steel specimen was also tested with corrosion marks, which were covered with paint. During testing, the coil generates eddy currents that automatically moves along the sample at a constant speed. Changes in temperature field on the sample surface were recorded with a thermal camera FLIR SC 7600. The eddy current stimulation was performed with a frequency of $35 \mathrm{kHz}$ and a power range from 80 to $150 \mathrm{~W}$ (maximum allowed power, $3 \mathrm{~kW}$ ).

Experimental research was carried out using the one-sided method, in which the source of thermal stimulation as well as thermal camera during testing are on the same side of the tested sample.

Figures 6 and 7 show sample experimental testing results. The thermogram (Figure 6) shows changes of the temperature field on the surface of a steel sample after undergoing scanning pulsed eddy current thermography. Figure 7 presents temperature changes on the surface of the sample shown in the thermogram (Fig. 6). Both the graph and the thermogram are clearly visible in the layers of different thicknesses. As shown in Figure 7, the increase in temperature signal is proportional to the number of layers of paint covering the surface of the metal.

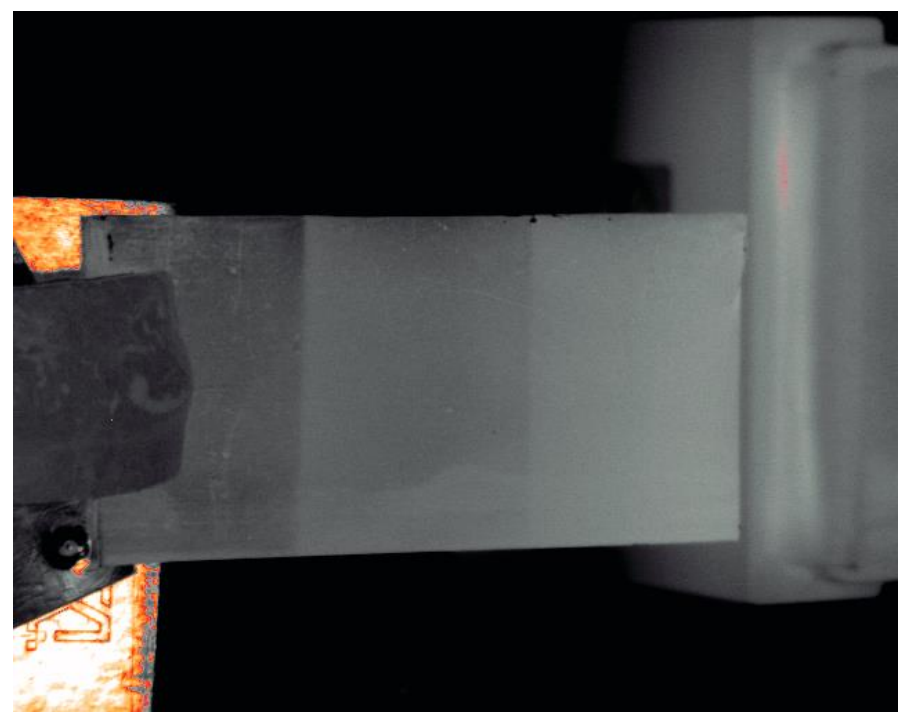

Fig. 6. Thermogram of changes of temperature field on the sample surface made of steel via scanning pulsed eddy current thermography. 


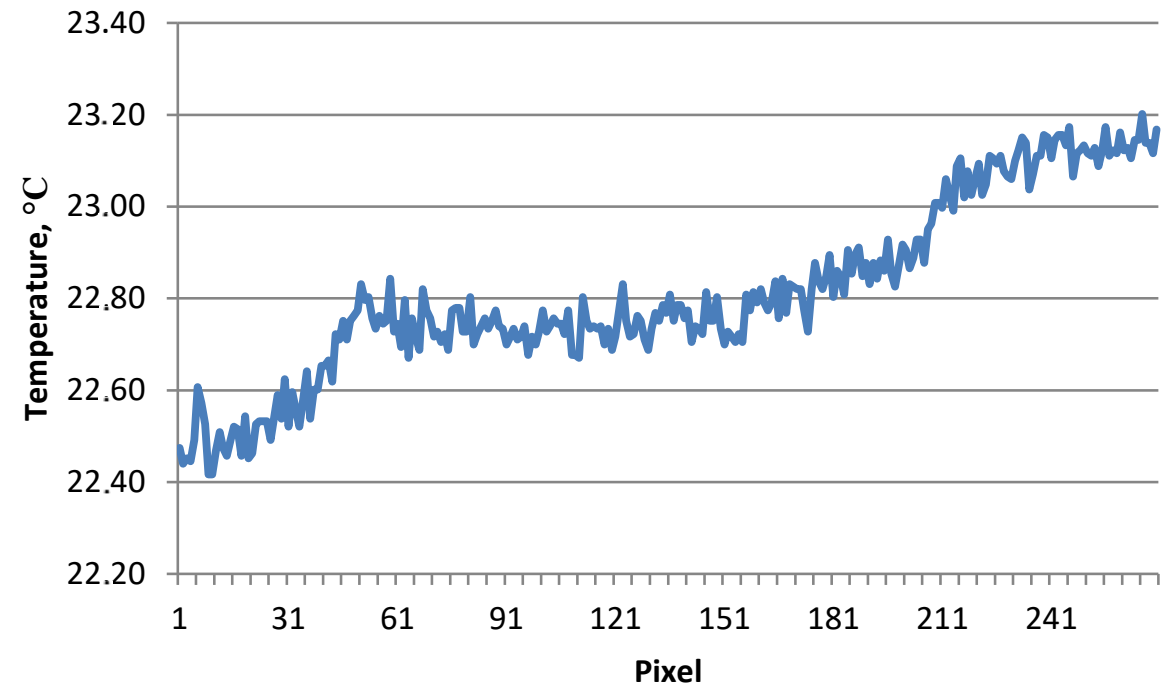

Fig. 7. The signal temperature changes on the surface of the sample (Figure 6) along its length.

Convergent results were also obtained when testing samples made of copper and aluminum. Clearly, also in graphs of temperature changes on the surface of samples after heating with eddy currents, there are places where there is a difference in number of layers of paint covering the surface of the tested samples. Figures 8 and 9 show graphs of temperature changes for these samples.

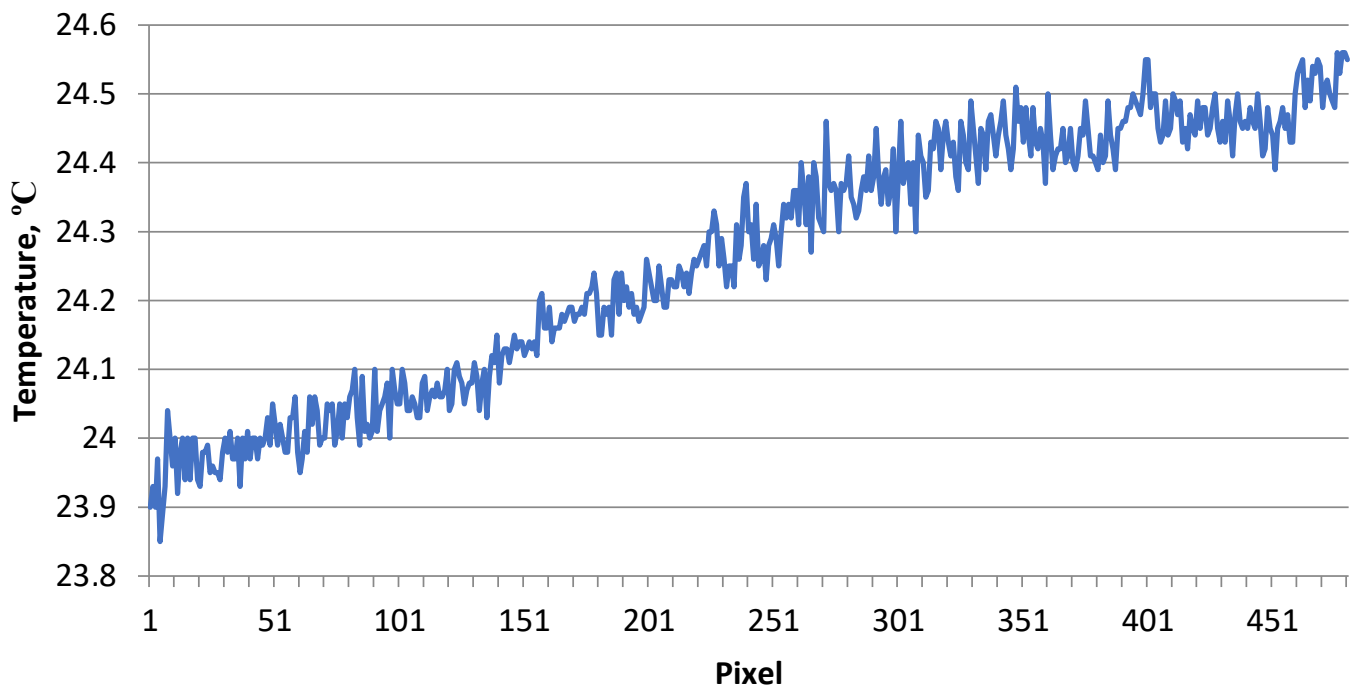

Fig. 8. The signal temperature changes on the surface of aluminum sample along its length. 


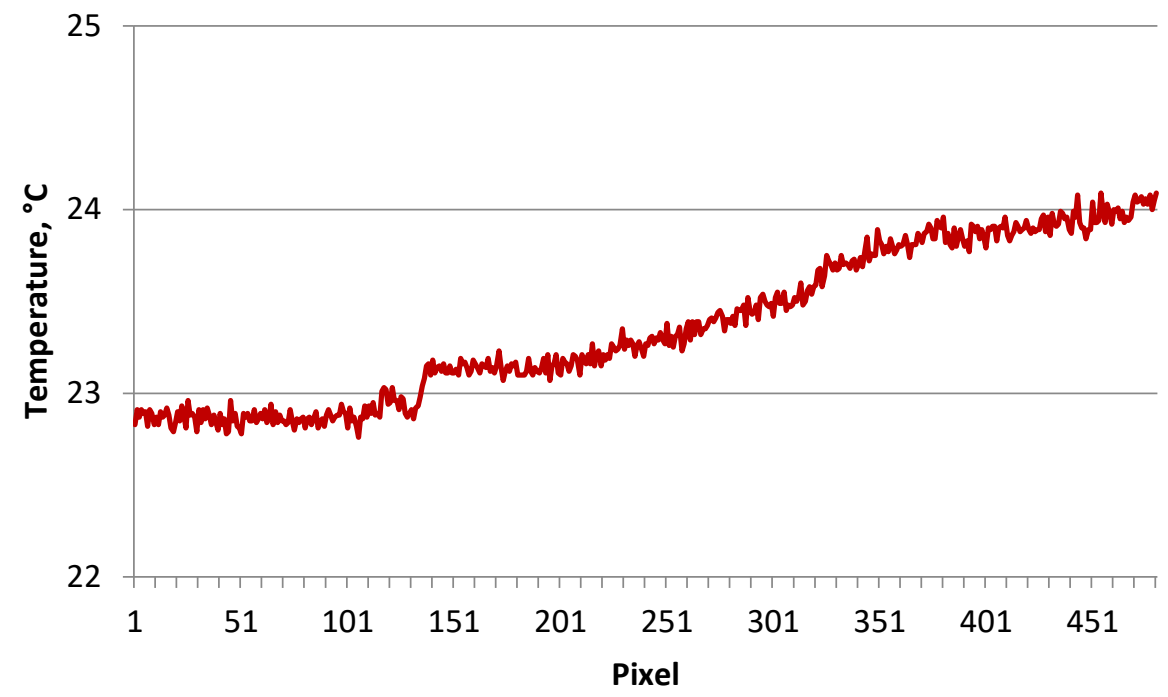

Fig. 9. The signal temperature changes on the surface of copper sample along its length

\section{Summary}

The experimental tests show that scanning pulsed eddy current thermography can be used to detect inconsistencies in the applied protective coating layer (paint, varnish). The method allows rapid evaluation of the surface protection layer of metal.

Differences between computer simulations and experimental results are caused by the following factors:

- in simulations, the distribution of generated heat was in the form of a heating line with uniform heat distribution, in experimental tests a moving probe was used, where the heat distribution isn't uniform;

- $\quad$ the thickness of the applied paint layer on the surface of the samples used in the experimental tests was not perfectly uniform, as was assumed in simulations;

- $\quad$ also, the painted surface of samples was not perfectly smooth;

- $\quad$ assumed heating power in simulations could differ from real power used in experiments;

- $\quad$ the thermophysical properties of the metals and paints in the simulations were selected on the basis of literature data and could differ from real properties.

Despite these differences, it can clearly be seen, on the basis of the obtained results, that there are very similar characteristics of changes in the temperature field on the surface of the tested samples obtained in both computer simulations and experimental tests.

\section{REFERENCES}

[1] Zenzinger G, Bamberg J, Satzger W, Carl V., "Thermographic Crack Detection by Eddy Current Excitation". Journal Nondestructive Testing and Evaluation, 22 (2-3): pp. 101-111, 2007

[2] Swiderski W., "Possibility of Defects Detection by Eddy Current Thermography Method in Marine Structures". Scientific Journals of the Maritime University of Szczecin, 44 (116): pp. 43-46: 2015.

[3] Swiderski W., "Detecting defects in marine structures by using eddy current infrared thermography". Applied Optics. 55 (34): D17-D21. 2016

[4] Kiran Kumar Ch.B., Krishnamurthy C.V., Maxfield B.W., Balasubramaniam K., "Tone Burst Eddy-Current Thermography (TBET)". Review of Quantitative Nondestructive Evaluation. American Institute of Physics, Vol. 27: pp. 544-51, 2008

[5] Maldague X., Nondestructive evaluation of materials by infrared thermography. London: Springer; 1993.

[6] Vavilov V.P., Burleigh D.D., "Review of pulsed thermal NDT: physical principles, theory and data processing". NDT \& E Int., Vol. 73: pp. 28-52, 2015

[7] ThermoEdCur, Operational manual. Innovation Ltd., 2016

[8] Panas A.J., Stryczniewicz W., Szczepaniak R., "Investigation of thermophysical properties of thin-layered paint". Thermochimica Acta, Vol. 662: pp. 100-107, 2018 